Keywords: dietary exposure; polychlorinated biphenyls; endocrine disrupting chemicals; environmental carcinogens breast cancer; endometrial cancer; ovary cancer; prospective cohort study

\title{
Dietary exposure to polychlorinated biphenyls and risk of breast, endometrial and ovarian cancer in a prospective cohort
}

\author{
Carolina Donat-Vargas ${ }^{1,2,3}$, Agneta Åkesson ${ }^{1}$, Marika Berglund ${ }^{1}$, Anders Glynn ${ }^{4}$, Alicja Wolk ${ }^{1}$ \\ and Maria Kippler ${ }^{\star}, 1$ \\ ${ }^{1}$ Institute of Environmental Medicine, Karolinska Institutet, Box 210, SE-171 77 Stockholm, Sweden; ${ }^{2}$ Department of Preventive \\ Medicine and Public Health, University of Navarra, 31008 Pamplona, Spain; ${ }^{3}$ IDISNA, Instituto de Investigación Sanitaria de \\ Navarra, 31008 Pamplona, Spain and ${ }^{4}$ Department of Risk and Benefit Assessment, National Food Agency, Box 622, SE-751 26 \\ Uppsala, Sweden
}

Background: Observational studies on polychlorinated biphenyl (PCB) exposure and hormone-related cancer risk are either inconsistent or lacking. We aimed to assess associations of dietary PCB exposure with breast, endometrial and ovarian cancer risk in middle-aged and elderly women.

Methods: We included 36777 cancer-free women at baseline in 1997 from the prospective population-based Swedish Mammography Cohort. Validated estimates of dietary PCB exposure were obtained via a food frequency questionnaire. Incident cancer cases were ascertained through register linkage.

Results: During 14 years of follow-up, we ascertained 1593, 437 and 195 incident cases of breast, endometrial and ovarian cancer. We found no overall association between dietary PCB exposure and any of these cancer forms. The multivariable-adjusted relative risks comparing women in the highest and lowest tertile of PCB exposure were 0.96 ( $95 \%$ confidence interval (Cl): $0.75,1.24), 1.21$ (95\% Cl: $0.73,2.01)$ and $0.90(95 \% \mathrm{Cl}: 0.45,1.79)$ for breast, endometrial and ovarian cancer. In analyses stratified by factors influencing oestrogen exposure, possibly masking associations with PCBs, indications of higher risks were observed for endometrial cancer.

Conclusions: This study suggests that dietary exposure to PCBs play no critical role in the development of breast, endometrial or ovarian cancer during middle-age and old ages.

Polychlorinated biphenyls (PCBs), comprising 209 synthetic congeners, have been extensively used in industrial and commercial products from the 1930s until the use was banned in most countries in the 1980s. Because PCBs are highly persistent and lipophilic, they are still widespread in the environment, where they bioaccumulate and magnify along the food chain. Consequently, humans are mainly exposed through food, in particular foods of animal origin such as fatty fish from contaminated waters (Bergkvist et al, 2012; Malisch and Kotz, 2014). In the body, PCBs are readily absorbed, distributed and stored in adipose tissue with half-lives of up to 15 years. Thus, elevated concentrations of PCBs are still found in the majority of the general population worldwide (CDC, 2009).

In 2013, PCBs were classified as human carcinogens by the International Agency for Research on Cancer based on evidence of excessive risk of melanoma in both occupationally exposed and in the general population (Lauby-Secretan et al, 2013; IARC, 2015). Although an increased risk of breast cancer as a result of PCB exposure is considered biologically plausible, due to $\mathrm{PCB}$ endocrine disrupting properties, including oestrogen-like activity

*Correspondence: Dr M Kippler; E-mail: maria.kippler@ki.se

Received 7 May 2016; revised 15 July 2016; accepted 15 August 2016; published online 15 September 2016

(c) 2016 Cancer Research UK. All rights reserved 0007-0920/16 
(Fang et al, 2000; Kester et al, 2000), the evidence is too limited to draw any conclusions. High levels of PCBs have been found in human breast tissue (Ellsworth et al, 2015) and experimental studies have suggested a role of PCBs in the aetiology of mammary tumour formation (Liu et al, 2010; Ptak et al, 2010). However, the observational data based on PCB biomarkers, either from case-control or nested-case-control studies, are partly inconsistent (Zhang et al, 2015; Leng et al, 2016). Some studies have observed a positive association, most often only with specific PCB congeners (Leng et al, 2016), certain groups of PCB congeners (Zhang et al, 2015), or in population sub-group analyses (Millikan et al, 2000), while others have observed no association.

Simultaneously, the capacity of PCBs to disrupt hormonedependent pathways, including steroid hormone systems, raise concern about a potential link with other female hormonesensitive cancers such as endometrial and ovarian cancer. Endometrial cancer has a relatively brief latency and is more sensitive towards exogenous and endogenous oestrogen than breast cancer (Adami et al, 1995; Akhmedkhanov et al, 2001). Still, only two case-control studies have explored the association of serum PCBs with endometrial cancer, without observing any significant association (Sturgeon et al, 1998; Weiderpass et al, 2000). To our knowledge, there is currently no published data on background exposure to PCBs and ovarian cancer. In electrical capacitor-manufacturing workers no clear increased mortality was observed for ovarian and uterine cancer (Ruder et al, 2014). Therefore, the aim of the present study was to prospectively assess the association between validated estimates of dietary PCB exposure and the risk of breast, endometrial and ovarian cancer in a large population-based cohort of middle-aged and elderly Swedish women.

\section{MATERIALS AND METHODS}

Study population. The Swedish Mammography Cohort, a population-based prospective cohort of women, was established during a mammography-screening programme between 1987 and 1990 (Harris et al, 2013). The source population, consisting of 90303 women born between 1914 and 1948, and residing in central Sweden (Uppsala and Västmanland counties), received a mailed self-administered questionnaire on diet and lifestyle (response rate $74 \%)$. In 1997, a second extended questionnaire was sent to all cohort members who were still alive and living in the study area (response rate 70\%; $n=39227$ ). The 1997 questionnaire was used as the baseline questionnaire in the present study, as there was sufficient documentation of the PCB content in different foods at this time (Bergkvist et al, 2012). Return of a complete questionnaire was considered as informed consent to participate in the study which has been approved by the Regional Ethical Review Board, Stockholm, Sweden (Harris et al, 2013).

For assessing the association of dietary PCB exposure and risk of breast, endometrial and ovarian cancer, we excluded women with an incorrect or missing personal identification number $(n=243)$, prevalent cancer at baseline $(n=1717)$ or implausible energy intake ( \pm 3 s.d.'s of mean log-transformed energy intake, $n=490$ ). Hence, the final study cohort consisted of 36777 women at start of follow-up. For the assessment of endometrial and ovarian cancer we additionally excluded women with hysterectomy $(n=504)$ or bilateral oophorectomy $(n=2637)$, respectively, at baseline (Figure 1$)$.

Questionnaire on diet and lifestyle factors. The baseline questionnaire included a 96-item food frequency questionnaire (FFQ), constructed to reflect the women's average consumption of

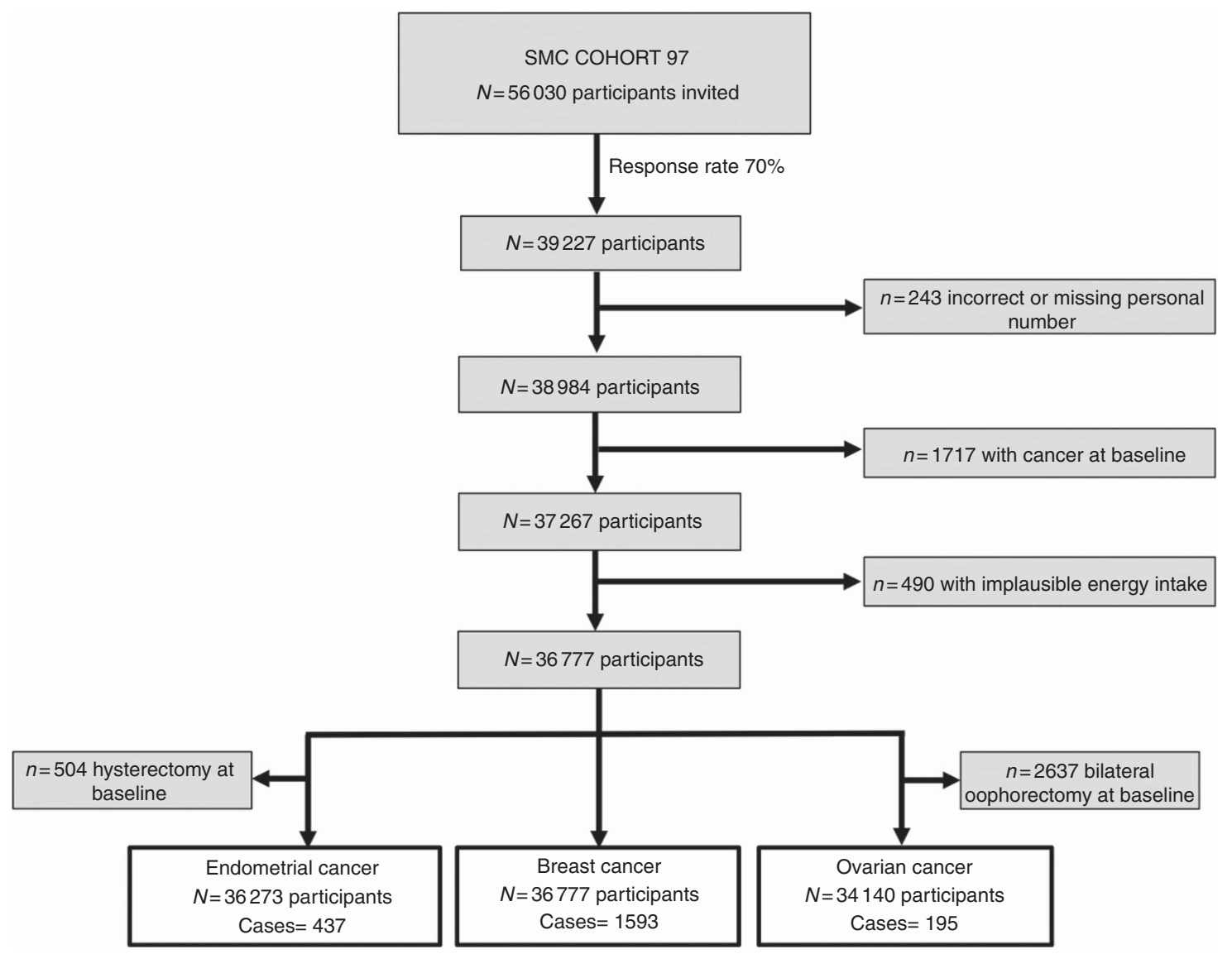

Figure 1. Flow chart of exclusion from the Swedish Mammography Cohort. 
different foods and beverages during the last year. For frequently consumed foods, the FFQ contained open-ended questions with pre-specified serving sizes, whereas for other foods it had eight predefined frequency categories ranging from never to three times per day. Age-specific portion-sizes were estimated from 5922 weighted food records kept by 213 randomly selected women from the study area. The FFQ has been validated, obtaining Pearson correlation coefficients ( $r$ ) between the average of four 1-week dietary records and the dietary questionnaire of 0.6 for fatty fish and $0.5-0.7$ for dairy products (Wolk A, personal communication).

The questionnaire also included questions on history of certain diseases and medications (oral contraceptives and postmenopausal hormones), as well as on level of education, body weight, height, age at menarche, parity, age at menopause, smoking habits and physical activity. Body mass index (BMI) was calculated as the weight in kilograms $(\mathrm{kg})$ divided by the square of the height in metres (m). The validity of BMI based on self-reported weight and height in the Swedish population has been shown to be high ( $r=0.85$; Kuskowska-Wolk et al, 1989). Women who did not report their menopausal status were classified as postmenopausal if they had gone through bilateral oophorectomy, used postmenopausal hormones, or were 55 years of age or older ( 95\% of the women reported to have entered menopause before 55 years of age). Physical activity was estimated using a validated questionnaire from which we obtained information about leisure-time activity (less or more than 2 hours of watching TV or sitting per day) and time spent walking or bicycling (less or more than 40 minutes per day; Orsini et al, 2008).

Assessment of dietary PCB exposure and other dietary factors. The dietary exposure to PCBs was estimated at the time of baseline through an extensive recipe-based database created for the FFQ, described in detail elsewhere (Bergkvist et al, 2012). The database was based on concentrations of the PCB-153 congener, which is the most abundant congener in food on the Swedish market and therefore a very good indicator of total PCBs, dioxin-like PCBs and the related polychlorinated dibenzodioxins and polychlorinated dibenzofurans in food and in human serum (Covaci et al, 2002; Bergkvist et al, 2012). The content of PCBs and long-chain omega3 fish fatty acids, such as eicosapentaenoic acid (EPA) and docosahexaenoic acid (DHA), in foods was obtained from the Swedish Food Database provided by the Swedish National Food Agency.

Daily dietary exposure to PCBs (ng per day) and dietary intake of EPA-DHA (mg per day) was estimated by multiplying the average concentration in various foods with the respective consumption frequency and portion size, and then, adjusting for total energy intake (mean of $1700 \mathrm{kcal}$ per day, for the cohort) using the residual-regression method (Willett and Stampfer, 1986). The FFQ-based dietary estimate of PCB exposure has been extensively validated against six serum PCB congeners $(118,138$, $153,156,170$ and 180) in a representative subsample of the cohort (Spearman correlation coefficients ranged from 0.30 to 0.58 ; Bergkvist et al, 2012). The FFQ-based dietary intake of EPA-DHA has been validated against adipose tissue concentrations; $r$ of 0.32 and 0.48 , respectively for concurrent, and 0.21 and 0.33 , respectively for past exposure assessment (6 years before the adipose tissue sampling) in women (Wallin et al, 2014).

Ascertainment of outcomes. Incident cases of invasive breast cancer, endometrial adenocarcinoma and epithelial ovarian cancer were ascertained by computerised linkage via the personal identification number to the national and regional Swedish Cancer Registers, which is close to $100 \%$ complete (Mattsson and Wallgren, 1984). For breast cancer, information about oestrogen receptor (ER) status of the tumour was obtained from the Quality Register at the Regional Oncology Centre in Uppsala. Information about oophorectomies and hysterectomies was obtained from the
National Patient Register. Ascertainment of deaths was also obtained through register linkage.

Statistical analyses. The women were followed from mid-September 1997 until the date of diagnosis of breast, endometrial or ovarian cancer, hysterectomy or bilateral oophorectomy (only for endometrial and ovarian cancer, respectively), death, or end of follow-up (31 December 2012), whichever occurred first. Women were categorised into tertiles of dietary PCB exposure at baseline. Hazard ratios (herein referred to as relative risks, RRs) and 95\% confidence intervals (CI) were estimated using Cox proportional hazard regression models with attained age (1-year units) as the underlying timescale. The proportional hazard assumption was checked by evaluating Schoenfeld's residuals and no departure from this assumption was observed. The models were adjusted for: postsecondary education (no/yes), family history of breast cancer (no/yes), oophorectomy (only for breast and endometrial cancers), history of diabetes (no/yes), BMI $\left(<18.5,18.5-25,25-30,>30 \mathrm{~kg} \mathrm{~m}^{-2}\right)$, weight loss $>5 \mathrm{~kg}$ within 1 year (no/yes), age at menarche $(<12$, $13, \geqslant 14$ years), ever use of oral contraceptives (no/yes), parity (no child, $1-2, \geqslant 3$ children), age at first birth (nulliparous, $<26$, $26-30, \geqslant 30$ years), age at menopause (premenopausal, $<51, \geqslant 51$ years), ever use of postmenopausal hormones (no/yes), smoking habits (current, former, never), leisure-time inactivity (high/low) and daily walking/cycling (low/high), alcohol consumption (no use, $0.1-5,5.1-15,>15 \mathrm{~g}$ per day) and total energy intake (continuous, kcal per day). We additionally adjusted for dietary EPA-DHA intake (tertiles, mg per day; Zheng et al, 2013). To test for linear trends across increasing categories of dietary $\mathrm{PCB}$ exposure we assigned the median exposure within each category and included it as a continuous variable.

We carried out a sensitivity analysis replicating the models after exclusion of premenopausal women at baseline $(<10 \%)$. To explore whether any potential PCB-related oestrogenic effect on breast and endometrial cancer development was masked by factors affecting the exogenous or endogenous oestrogen exposure, we stratified by BMI $\left(\leqslant 25,25-30,>30 \mathrm{~kg} \mathrm{~m}^{-2}\right.$ (for endometrial cancer only $\leqslant 25$ and $>25 \mathrm{~kg} \mathrm{~m}^{-2}$ ); Akhmedkhanov et al, 2001), ever use of postmenopausal hormones (no/yes; Reeves et al, 2006) and smoking status (never/ever; Terry et al, 2002). Stratified analyses were not conducted for ovarian cancer due to the limited number of cases $(n=195)$. We tested for interactions on the multiplicative scale using the likelihood ratio test, comparing models with and without an interaction term. In addition, as endometrial cancer seems to be particularly sensitive to hormonal factors (Adami et al, 1995), in order to avoid as much as possible that the effect of PCBs was disguised by other factors affecting the oestrogen exposure, we conducted the analyses excluding women who were overweight and obese $\left(\mathrm{BMI}>25 \mathrm{~kg} \mathrm{~m}^{-2}\right)$, ever users of postmenopausal hormones and ever smokers.

Finally, based on recent experimental evidence showing that prenatal exposure to the polychlorinated organic pollutant dioxin (2,3,7,8-tetrachlorodibenzo-p-dioxin; same dietary sources as PCBs) doubled mammary tumour incidence only in mice fed an obesity-associated diet high in animal fat (La Merrill et al, 2010), we conducted stratified analysis among non-obese and obese women $\left(\mathrm{BMI} \leqslant 30\right.$ or $>30 \mathrm{~kg} \mathrm{~m}^{-2}$ ) consuming a diet either low or high in saturated fat (median split). Missing values were generally below $5 \%$ for the covariates, with the exception of age at menopause (13\%). We fitted the multivariable-adjusted models including the missing values in a separate category. The results did, however, not differ after replicating the main models using a multiple imputation chained equation technique with 20 imputations to handle missing data. All $P$-values presented were two-tailed, and $P$-values $<0.05$ were considered statistically 
significant. Analyses were performed using STATA/SE version 13.0 (StataCorp LP, College Station, TX, USA).

\section{RESULTS}

During 14 years of follow-up (505623 person-years), we ascertained 1593, 437 and 195 incident cases of breast, endometrial and ovarian cancer. Information on ER status was available in $83 \%$ of the breast cancer cases out of which 1155 were ER + and 162 were ER - . The mean age at diagnosis was 69,71 and 69 years for breast, endometrial and ovarian cancer, respectively. The median dietary PCB exposure was 162 ng per day (5-95th percentile: 70$368 \mathrm{ng}$ per day). No major differences in baseline age-standardised characteristics were observed across tertiles of dietary PCB exposure, with the exception of dietary intake of EPA-DHA, which was about three times higher among women in the highest tertile of dietary PCB exposure compared with those in the lowest tertile (Table 1).

We observed no significant association between dietary PCB exposure and breast cancer risk in either the age- or multivariableadjusted model (Table 2). The RR of the fully adjusted model (additionally adjusted for dietary intake of EPA-DHA) was 0.96 (95\% CI: $0.75,1.24)$ when comparing women in the highest tertile of dietary PCB exposure with those in the lowest. Likewise, no associations were observed in relation to ER + and ER - breast cancer. No association was observed between dietary PCB exposure and endometrial or ovarian cancer; comparing women in the highest with those in the lowest tertile of dietary PCB exposure, the fully multivariable-adjusted RR was 1.21 (95\% CI: $0.73,2.01)$ and 0.90 (95\% CI: $0.45,1.79$ ), respectively (Table 2). In sensitivity analysis, the corresponding fully multivariable-adjusted RR for the three cancers after excluding premenopausal women at baseline was $1.00(95 \% \mathrm{Cl}: 0.77,1.30 ; 1445$ cases of breast cancer), 1.21 (95\% Cl: $0.71,2.05 ; 400$ cases of endometrial cancer) and 1.06 (95\% Cl: $0.51,2.20 ; 174$ cases of ovarian cancer).

To limit the potential impact of factors affecting the variation in endogenous and exogenous oestrogen exposure, which might obscure any relationship between dietary PCB exposure and cancer risk, we stratified by BMI, ever use of postmenopausal hormones, and smoking status. Comparing women in the highest and lowest tertile of dietary PCB exposure, the fully multivariable-adjusted $\mathrm{RR}$ for breast cancer differed slightly by BMI, but not by postmenopausal hormone use or smoking status ( $P$ for interaction $>0.3$ for all; Table 3). Accordingly, the RR for lean women was 0.81 (95\% Cl: $0.58,1.14)$, for overweight women was $1.11(95 \% \mathrm{Cl}$ : $0.73,1.71)$ and for obese women was 1.32 (95\% Cl: 0.61, 2.85).

For endometrial cancer, the corresponding fully multivariableadjusted RR for dietary PCBs was higher among lean women (RR 1.34; 95\% CI: 0.60, 2.98) and even higher among never users of postmenopausal hormones (RR 1.57; 95\% CI: 0.75, 3.32), but the association only reached statistical significance among never smokers (RR 2.02; 95\% CI: 1.06, 3.86; Table 4) without evidence of any interactions ( $P$ for interaction $>0.3$ for all). When we excluded women who were overweight and obese, ever used postmenopausal hormones and ever smoked, the multivariableadjusted RR among the remaining women was 2.42 (95\% Cl: 0.43 , 13.73) when comparing women in the highest tertile of dietary PCB exposure with those in the lowest.

Finally, the RRs for breast cancer when we conducted a stratified analysis among non-obese and obese women $(\mathrm{BMI} \leqslant 30$ or $>30 \mathrm{~kg} \mathrm{~m}^{-2}$ ) consuming a diet either low or high in saturated fat were $0.91(95 \% \mathrm{Cl}: 0.30,2.73)$ for obese women with a low saturated fat intake and $2.19(95 \% \mathrm{Cl}: 0.73,6.54)$ for obese women with a high saturated fat intake, comparing women in the highest and lowest tertile of dietary PCB exposure (Figure 2). No evidence of an interaction was observed between saturated fat intake and $\mathrm{PCB}$ exposure $(P$ for interaction $=0.96)$.

\section{DISCUSSION}

It is biologically plausible that hormonally active chemicals, including PCBs, contribute to the risk of cancers in hormonesensitive tissues such as the breast, endometrium and ovary. In this large prospective cohort of Swedish women, we observed, however,

Table 1. Age-standardised baseline characteristics of 36777 women from the Swedish Mammography Cohort by tertiles of dietary PCB exposure

\begin{tabular}{|c|c|c|c|}
\hline Tertiles of dietary PCB exposure range (median) ng per day ${ }^{a}$ & $<139(110)$ & $139-193(162)$ & $>193(256)$ \\
\hline Age (years) & $62 \pm 10$ & $60 \pm 9$ & $63 \pm 9$ \\
\hline Family history of breast cancer (\%) & 9 & 9 & 9 \\
\hline History of diabetes (\%) & 5 & 4 & 6 \\
\hline Weight loss $>5 \mathrm{~kg}$ within 1 year (\%) & 68 & 69 & 70 \\
\hline Age at menarche $\leqslant 12$ years (\%) & 28 & 27 & 29 \\
\hline Ever use of oral contraceptives (\%) & 55 & 58 & 58 \\
\hline Premenopausal (\%) & 11 & 12 & 11 \\
\hline Age at menopause $\geqslant 51$ years $(\%)$ & 42 & 43 & 43 \\
\hline Ever use of postmenopausal hormones (\%) & 50 & 52 & 51 \\
\hline Current smoker (\%) & 24 & 22 & 24 \\
\hline \multicolumn{4}{|l|}{ Leisure-time daily physical activity (\%) } \\
\hline$\leqslant 2 \mathrm{~h}$ sitting/watching TV & 56 & 55 & 56 \\
\hline$>40$ min walking/bicycling & 36 & 35 & 36 \\
\hline
\end{tabular}


Table 2. RR ( $95 \% \mathrm{Cls})$ of breast, endometrial and ovarian cancer by tertiles of dietary PCB exposure

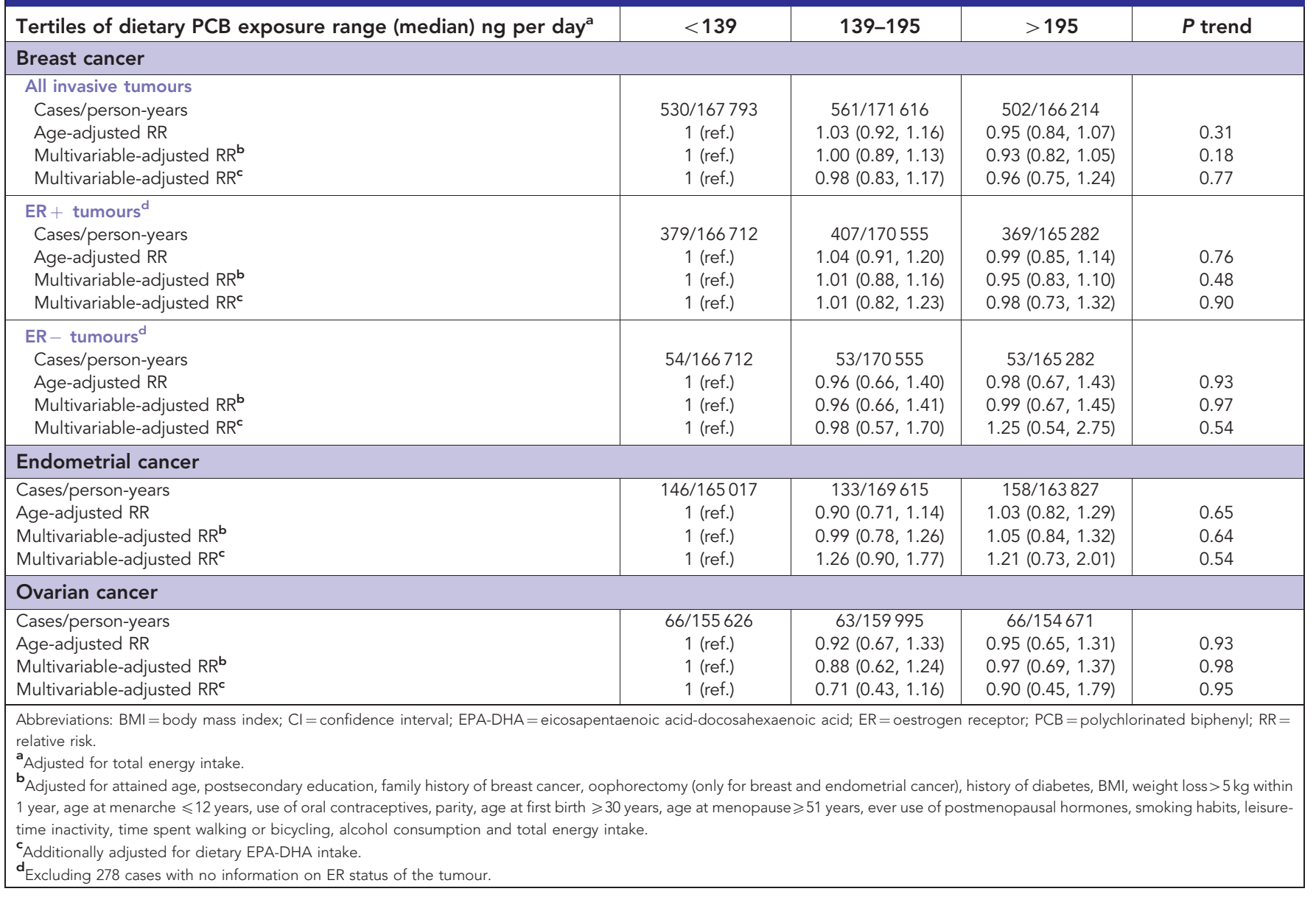

no indication of an overall association between validated estimates of dietary PCB exposure and incident breast, endometrial or ovarian cancer. To account for variations in exogenous and endogenous oestrogen exposures potentially obscuring any associations, we stratified by adiposity, postmenopausal hormone use and smoking status. Focusing on breast cancer, we observed no association among women expected to be less influenced by oestrogen exposures (lean, never users of postmenopausal hormones and never smokers). For the more oestrogen sensitive endometrial cancer, indications of higher risks were suggested in these three groups of women, but statistical significance was only reached among never smokers.

Our null results on breast cancer risk are in accordance with the majority of the previous case-control (Zheng et al, 2000; Gammon et al, 2002; Lopez-Carrillo et al, 2002; Rubin et al, 2006; Gatto et al, 2007) and nested-case-control studies (Wolff et al, 1993; Krieger et al, 1994; Helzlsouer et al, 1999; Ward et al, 2000; Wolff et al, 2000; Laden et al, 2001; Raaschou-Nielsen et al, 2005), observing no association between either total PCB exposure-measured in adipose tissue or blood-or between specific congeners (including PCB-153) and risk of breast cancer. In addition, two meta-analyses found no significant association with total PCB exposure when comparing the highest and lowest catesgories (odds ratio $=1.15$, 95\% CI: 0.92, 1.43; $I^{2}=70.6 \%$ (Zani et al, 2013) and odds ratio $=1.09,95 \%$ CI: 0.97, 1.22; $I^{2}=55.4 \%$ (Zhang et al, 2015)).

Although several studies have reported significant associations between specific PCB congeners and breast cancer, the pooled OR from congener-specific meta-analysis were only statistically significant for PCB-99, PCB-183 and PCB-187 (Leng et al, 2016). Nevertheless, the results are difficult to interpret due to multiple comparisons, as well as the high correlation between different congeners, resulting in collinearity. Moreover, when congeners are assessed individually, the potential additive or synergistic effects are not taken into account. By grouping PCBs according to their structural, biological and pharmacokinetics properties, Zhang et al (2015), observed significant pooled ORs for PCB group II (potentially anti-oestrogenic and immunotoxic, dioxin-like) and group III (phenobarbital, CYP1A and CYP2B inducers, biologically persitent), but not for group I (potentially oestrogenic) (Zhang et al, 2015).

Interestingly, in an experimental study on early-life exposure, the polychlorinated organic pollutant dioxin induced mammary tumour incidence in obese mice depending on whether the animals were fed a diet high in saturated fat or not (La Merrill et al, 2010). In line with this, we observed a statistically non-significant increased breast cancer risk with increasing long-term dietary PCB exposure among obese women with a high intake of saturated fat, while no association was observed among obese women with a low intake of saturated fat or among the non-obese women. Any potential mechanism behind this finding remains to be elucidated.

Although our study is the first one to explore an association between background exposure to PCBs and ovarian cancer risk, our null findings for endometrial cancer are in accordance with two other previous population-based case-control studies (Sturgeon et al, 1998; Weiderpass et al, 2000). In the study by Weiderpass and coworkers, which included 154 cases and 205 controls residing in Sweden, no association was observed between quartiles of total PCBs (10 congeners) in serum and endometrial cancer (OR 1.2; 95\% CI: 0.6, 2.2; Weiderpass et al, 2000). They also 
Table 3. RR $(95 \% \mathrm{Cls})$ of breast cancer according to tertiles of dietary PCB exposure stratified by BMI, use of postmenopausal hormones and smoking status

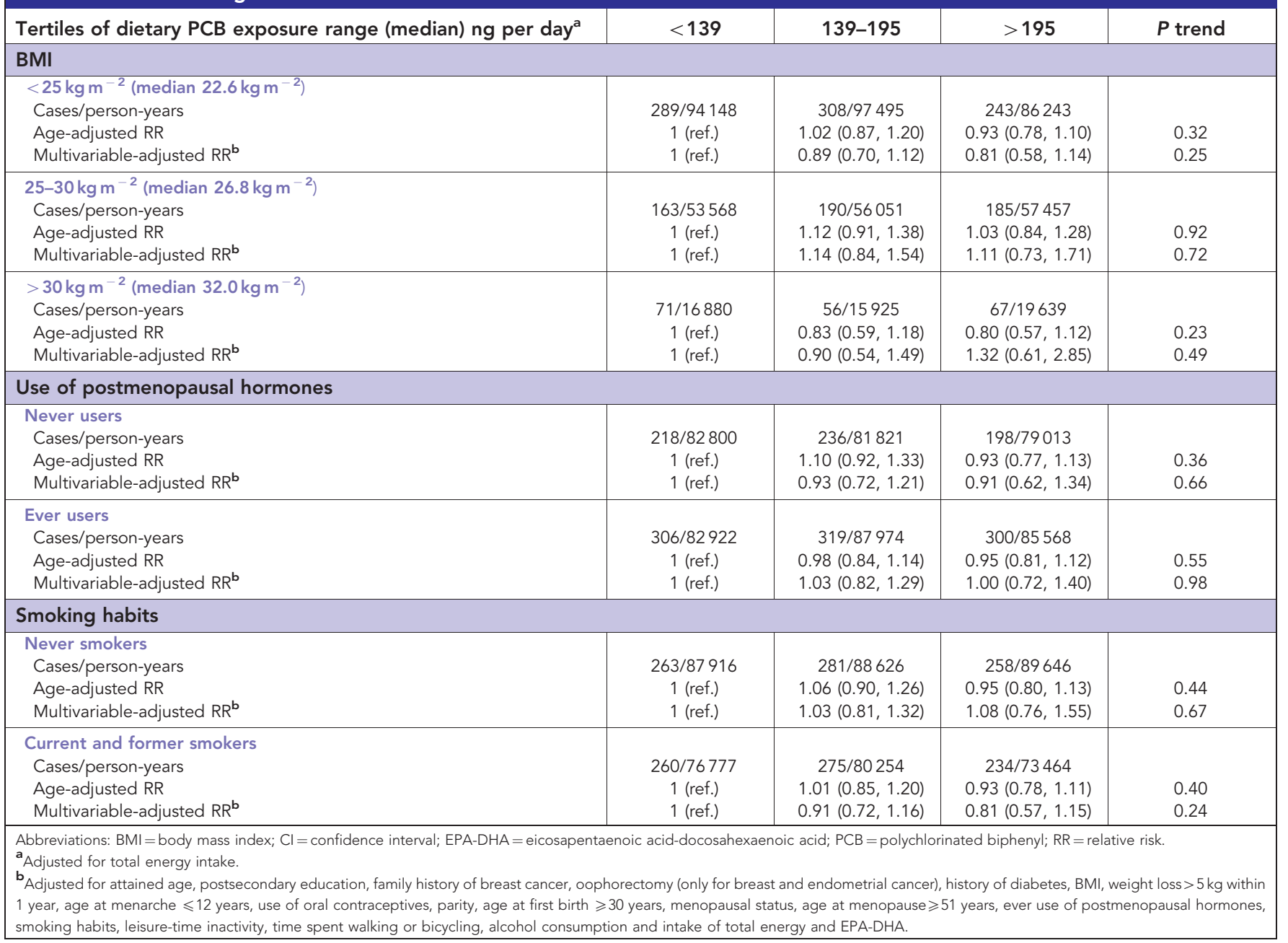

observed no significant associations for individual congeners (e.g., for PCB-153 the OR was 0.9 (95\% CI: $0.5,1.7)$ ) or congeners grouped by different hormonal activity. Similarly, the other study (Sturgeon et al, 1998), based on 90 cases and 90 controls from a multicenter study in five geographic regions in the US, obtained an OR of 0.9 (95\% Cl: 0.4 , 2.5) when comparing the highest quartile of total serum PCBs (in total 27 congeners assessed jointly) with the lowest quartile.

Our overall null-findings observed in this large populationbased prospective cohort could be explained by that PCBs, at the levels present in the general population, do not increase the risk of cancer in the breast, endometrium or ovary. Specific PCB congeners have been shown to exert opposite hormonal effects (e.g., oestrogenic, anti-oestrogenic or/and anti-androgenic (Fang et al, 2000; Fang et al, 2003; Zhang et al, 2015)), suggesting that the balance between these opposite responses to individual congeners may be the reason for the overall null findings. We did, however, observe a statistically significant positive dose-response association between dietary PCBs and endometrial cancer risk among never smokers, whereas no association was observed in smokers. Although we cannot exclude that this represents a chance finding, it can be speculated that tobacco smoking - which exerts anti-oestrogen effect via increased metabolic clearance of circulating oestrogen concentrations, a reduction in relative body weight, and an earlier age at menopause (Terry et al, 2002) - obscured the association between PCBs and endometrial cancer. Likewise, it can be speculated that the suggested higher endometrial cancer risk observed among lean women and never users of hormone replacement therapy, reflects masking of the associations by adiposity and postmenopausal hormone use.

The major strengths of our study include (i) its prospective population-based design, which avoids reverse causation bias and allows us to take into account long-term exposure to dietary PCBs, (ii) the large sample size with an ample number of cases, (iii) the almost $100 \%$ complete cancer ascertainment in the Swedish Cancer Registry (Mattsson and Wallgren, 1984), minimising differential loss to follow-up, (iv) the availability of detailed and validated data on PCBs and other dietary exposures and data on other potential risk factors for these female cancers, and (vi) that we took into account both the time-trend of decreasing PCB concentrations in food and the effect of the processing on the PCB concentrations in cooked food in the dietary PCB exposure estimation (Bergkvist et al, 2012). Potential limitations include the measurement error and subsequent misclassification of PCB exposure and the limited number of cases in some stratified analyses, which could influence the lack of observed associations. Although the diet is the major route of exposure to PCBs (>95\%) in the general population (Malisch and Kotz, 2014) and various dietary patterns have been associated with serum PCB levels (Ax et al, 2015), additional exposures from other non-dietary sources, such as indoor air PCBs, cannot be excluded. Exposure via inhalation in contaminated buildings constructed in 1950-70, may mainly contribute to the less chlorinated PCB congeners (Bräuner et al, 2016). Also, because it is not possible to discriminate between different contaminants present in the same foods as PCBs, we 


\section{CONFLICT OF INTEREST}

The authors declare no conflict of interest.

\section{REFERENCES}

Adami HO, Lipworth L, Titus-Ernstoff L, Hsieh CC, Hanberg A, Ahlborg U, Baron J, Trichopoulos D (1995) Organochlorine compounds and estrogen-related cancers in women. Cancer Causes Control 6(6): 551-566.

Akhmedkhanov A, Zeleniuch-Jacquotte A, Toniolo P (2001) Role of exogenous and endogenous hormones in endometrial cancer: review of the evidence and research perspectives. Ann N Y Acad Sci 943: 296-315.

Ax E, Lampa E, Lind L, Salihovic S, van Bavel B, Cederholm T, Sjögren P, Lind PM (2015) Circulating levels of environmental contaminants are associated with dietary patterns in older adults. Environ Int 75: 93-102.

Bergkvist C, Akesson A, Glynn A, Michaelsson K, Rantakokko P, Kiviranta H, Wolk A, Berglund M (2012) Validation of questionnaire-based long-term dietary exposure to polychlorinated biphenyls using biomarkers. Mol Nutr Food Res 56(11): 1748-1754.

Bräuner EV, Andersen ZJ, Frederiksen M, Specht IO, Hougaard KS, Ebbehøj N, Bailey J, Giwercman A, Steenland K, Longnecker MP, Bonde JP (2016) Health effects of pcbs in residences and schools (HESPERUS): PCB-health cohort profile. Sci Rep 19(6): 24571.

CDC (2009) Fourth national report on human exposure to environmental chemicals.

Covaci A, Koppen G, Van Cleuvenbergen R, Schepens P, Winneke G, van Larebeke N, Nelen V, Vlietinck R, Schoeters G (2002) Persistent organochlorine pollutants in human serum of 50-65 years old women in the flanders environmental and health study (flehs). Part 2: Correlations among pcbs, pcdd/pcdfs and the use of predictive markers. Chemosphere 48(8): 827-832.

Ellsworth RE, Mamula KA, Costantino NS, Deyarmin B, Kostyniak PJ, Chi LH, Shriver CD, Ellsworth DL (2015) Abundance and distribution of polychlorinated biphenyls (pcbs) in breast tissue. Environ Res 138 : 291-297.

Fang H, Tong W, Perkins R, Soto AM, Prechtl NV, Sheehan DM (2000) Quantitative comparisons of in vitro assays for estrogenic activities. Environ Health Perspect 108(8): 723-729.

Fang H, Tong W, Branham WS, Moland CL, Dial SL, Hong H, Xie Q, Perkins R, Owens W, Sheehan DM (2003) Study of 202 natural, synthetic, and environmental chemicals for binding to the androgen receptor. Chem Res Toxicol 16(10): 1338-1358.

Gammon MD, Wolff MS, Neugut AI, Eng SM, Teitelbaum SL, Britton JA, Terry MB, Levin B, Stellman SD, Kabat GC, Hatch M, Senie R, Berkowitz G, Bradlow HL, Garbowski G, Maffeo C, Montalvan P, Kemeny M, Citron M, Schnabel F, Schuss A, Hajdu S, Vinceguerra V, Niguidula N, Ireland K, Santella RM (2002) Environmental toxins and breast cancer on long island. Ii. Organochlorine compound levels in blood. Cancer Epidemiol Biomarkers Prev 11(8): 686-697.

Gatto NM, Longnecker MP, Press MF, Sullivan-Halley J, McKean-Cowdin R, Bernstein L (2007) Serum organochlorines and breast cancer: A casecontrol study among african-american women. Cancer Causes Control 18(1): 29-39.

Harris H, Håkansson N, Olofsson C, Julin B, Åkesson A, Wolk A (2013) The swedish mammography cohort and the cohort of swedish men: Study design and characteristics of two population-based longitudinal cohorts. OA Epidemiol 1(2): 16.

Helzlsouer KJ, Alberg AJ, Huang HY, Hoffman SC, Strickland PT, Brock JW, Burse VW, Needham LL, Bell DA, Lavigne JA, Yager JD, Comstock GW (1999) Serum concentrations of organochlorine compounds and the subsequent development of breast cancer. Cancer Epidemiol Biomarkers Prev 8(6): 525-532.

IARC (2015) Polychlorinated and polybrominated biphenyls. (IARC Monongraphs on the evalution of carcirongenic risks to humans).

Kester MH, Bulduk S, Tibboel D, Meinl W, Glatt H, Falany CN, Coughtrie MW, Bergman A, Safe SH, Kuiper GG, Schuur AG, Brouwer A, Visser T] (2000) Potent inhibition of estrogen sulfotransferase by hydroxylated pcb metabolites: A novel pathway explaining the estrogenic activity of pcbs. Endocrinology 141(5): 1897-1900.
Krieger N, Wolff MS, Hiatt RA, Rivera M, Vogelman J, Orentreich N (1994) Breast cancer and serum organochlorines: a prospective study among white, black, and asian women. J Natl Cancer Inst 86(8): 589-599.

Kuskowska-Wolk A, Karlsson P, Stolt M, Rossner S (1989) The predictive validity of body mass index based on self-reported weight and height. Int J Obes 13(4): 441-453.

La Merrill M, Harper R, Birnbaum LS, Cardiff RD, Threadgill DW (2010) Maternal dioxin exposure combined with a diet high in fat increases mammary cancer incidence in mice. Environ Health Perspect 118(5): 596-601.

Laden F, Hankinson SE, Wolff MS, Colditz GA, Willett WC, Speizer FE, Hunter DJ (2001) Plasma organochlorine levels and the risk of breast cancer: an extended follow-up in the nurses' health study. Int J Cancer 91(4): 568-574.

Lauby-Secretan B, Loomis D, Grosse Y, El Ghissassi F, Bouvard V, Benbrahim-Tallaa L, Guha N, Baan R, Mattock H, Straif K (2013) Carcinogenicity of polychlorinated biphenyls and polybrominated biphenyls. Lancet Oncol 14(4): 287-288.

Leng L, Li J, Luo XM, Kim JY, Li YM, Guo XM, Chen X, Yang QY, Li G, Tang NJ (2016) Polychlorinated biphenyls and breast cancer: a congenerspecific meta-analysis. Environ Int 88: 133-141.

Liu S, Li S, Du Y (2010) Polychlorinated biphenyls (pcbs) enhance metastatic properties of breast cancer cells by activating rho-associated kinase (rock). PLoS One 5(6): e11272.

Lopez-Carrillo L, Lopez-Cervantes M, Torres-Sanchez L, Blair A, Cebrian ME, Garcia RM (2002) Serum levels of beta-hexachlorocyclohexane, hexachlorobenzene and polychlorinated biphenyls and breast cancer in mexican women. Eur J Cancer Prev 11(2): 129-135.

Malisch R, Kotz A (2014) Dioxins and PCBs in feed and food-review from european perspective. Sci Total Environ 491-492: 2-10.

Mattsson B, Wallgren A (1984) Completeness of the swedish cancer register. Non-notified cancer cases recorded on death certificates in 1978. Acta Radiol Oncol 23(5): 305-313.

Millikan R, DeVoto E, Duell EJ, Tse CK, Savitz DA, Beach J, Edmiston S, Jackson S, Newman B (2000) Dichlorodiphenyldichloroethene, polychlorinated biphenyls, and breast cancer among african-american and white women in north carolina. Cancer Epidemiol Biomarkers Prev 9(11): 1233-1240.

Orsini N, Bellocco R, Bottai M, Hagstromer M, Sjostrom M, Pagano M, Wolk A (2008) Validity of self-reported total physical activity questionnaire among older women. Eur J Epidemiol 23(10): 661-667.

Ptak A, Ludewig G, Rak A, Nadolna W, Bochenek M, Gregoraszczuk EL (2010) Induction of cytochrome p450 1a1 in mcf-7 human breast cancer cells by 4-chlorobiphenyl (pcb3) and the effects of its hydroxylated metabolites on cellular apoptosis. Environ Int 36(8): 935-941.

Raaschou-Nielsen O, Pavuk M, Leblanc A, Dumas P, Philippe Weber J, Olsen A, Tjonneland A, Overvad K, Olsen JH (2005) Adipose organochlorine concentrations and risk of breast cancer among postmenopausal danish women. Cancer Epidemiol Biomarkers Prev 14(1): 67-74.

Reeves GK, Beral V, Green J, Gathani T, Bull D (2006) Hormonal therapy for menopause and breast-cancer risk by histological type: a cohort study and meta-analysis. Lancet Oncol 7(11): 910-918.

Rubin CH, Lanier A, Kieszak S, Brock JW, Koller KR, Strosnider H, Needham L, Zahm S, Harpster A (2006) Breast cancer among alaska native women potentially exposed to environmental organochlorine chemicals. Int $J$ Circumpolar Health 65(1): 18-27.

Ruder AM, Hein MJ, Hopf NB, Waters MA (2014) Mortality among 24865 workers exposed to polychlorinated biphenyls (pcbs) in three electrical capacitor manufacturing plants: a ten-year update. Int $J$ Hyg Environ Health 217(2-3): 176-187.

Sturgeon SR, Brock JW, Potischman N, Needham LL, Rothman N, Brinton LA, Hoover RN (1998) Serum concentrations of organochlorine compounds and endometrial cancer risk (united states). Cancer Causes Control 9(4): 417-424.

Terry PD, Rohan TE, Franceschi S, Weiderpass E (2002) Cigarette smoking and the risk of endometrial cancer. Lancet Oncol 3(8): 470-480.

Wallin A, Di Giuseppe D, Burgaz A, Hakansson N, Cederholm T, Michaelsson K, Wolk A (2014) Validity of food frequency questionnaire-based estimates of long-term long-chain n-3 polyunsaturated fatty acid intake. Eur J Nutr 53(2): 549-555.

Ward EM, Schulte P, Grajewski B, Andersen A, Patterson Jr DG, Turner W, Jellum E, Deddens JA, Friedland J, Roeleveld N, Waters M, Butler MA, 
DiPietro E, Needham LL (2000) Serum organochlorine levels and breast cancer: a nested case-control study of norwegian women. Cancer Epidemiol Biomarkers Prev 9(12): 1357-1367.

Weiderpass E, Adami HO, Baron JA, Wicklund-Glynn A, Aune M, Atuma S, Persson I (2000) Organochlorines and endometrial cancer risk. Cancer Epidemiol Biomarkers Prev 9(5): 487-493.

Willett W, Stampfer MJ (1986) Total energy intake: implications for epidemiologic analyses. Am J Epidemiol 124(1): 17-27.

Wolff MS, Zeleniuch-Jacquotte A, Dubin N, Toniolo P (2000) Risk of breast cancer and organochlorine exposure. Cancer Epidemiol Biomarkers Prev 9(3): 271-277.

Wolff MS, Toniolo PG, Lee EW, Rivera M, Dubin N (1993) Blood levels of organochlorine residues and risk of breast cancer. J Natl Cancer Inst 85(8): 648-652.

Zani C, Toninelli G, Filisetti B, Donato F (2013) Polychlorinated biphenyls and cancer: an epidemiological assessment. J Environ Sci Health C Environ Carcinog Ecotoxicol Rev 31(2): 99-144.
Zhang J, Huang Y, Wang X, Lin K, Wu K (2015) Environmental polychlorinated biphenyl exposure and breast cancer risk: a meta-analysis of observational studies. PLoS One 10(11): e0142513.

Zheng JS, Hu XJ, Zhao YM, Yang J, Li D (2013) Intake of fish and marine n-3 polyunsaturated fatty acids and risk of breast cancer: meta-analysis of data from 21 independent prospective cohort studies. BMJ 346: f3706.

Zheng T, Holford TR, Mayne ST, Tessari J, Ward B, Carter D, Owens PH, Boyle P, Dubrow R, Archibeque-Engle S, Dawood O, Zahm SH (2000) Risk of female breast cancer associated with serum polychlorinated biphenyls and 1,1-dichloro-2,2'-bis(p-chlorophenyl)ethylene. Cancer Epidemiol Biomarkers Prev 9(2): 167-174.

This work is published under the standard license to publish agreement. After 12 months the work will become freely available and the license terms will switch to a Creative Commons AttributionNonCommercial-Share Alike 4.0 Unported License. 